\title{
Rapid evaluation of 25 key sphingolipids and phosphosphingolipids in human plasma by LC-MS/MS
}

\author{
Abdul Basit • Daniele Piomelli • Andrea Armirotti \\ Received: 19 November 2014 / Revised: 13 February 2015 / Accepted: 20 February 2015 /Published online: 8 March 2015 \\ (C) The Author(s) 2015. This article is published with open access at Springerlink.com
}

\begin{abstract}
We report on a new, sensitive, and fast LCMS/MS method for the simultaneous determination of 25 key sphingolipid components in human plasma, including phosphorylated sphinganine and sphingosine, in a single 9-min run. This method enables an effective and high-throughput coverage of the metabolic changes involving the sphingolipidome during physiological or pathological states. The method is based on liquid-liquid extraction followed by reversed-phase LC-MS/MS. Exogenous odd-chain lipids are used as cost-effective but reliable internal standards. The method was fully validated in surrogate matrix and naive human plasma following FDA guidelines. Sample stability and dilution integrity were also tested and verified.
\end{abstract}

Keywords Ceramides · Sphingomyelins ·

Sphingosine-1-phosphate $\cdot$ Sphinganine-1-phosphate .

LC-MS/MS · Human plasma

Published in the topical collection Lipidomics with guest editor Michal Holčapek.

Electronic supplementary material The online version of this article (doi:10.1007/s00216-015-8585-6) contains supplementary material, which is available to authorized users.

A. Basit $\cdot$ D. Piomelli $\cdot$ A. Armirotti $(\square)$

Department of Drug Discovery and Development, Istituto Italiano di

Tecnologia, via Morego 30, 16163 Genoa, Italy

e-mail: Andrea.Armirotti@iiit.it

\section{Piomelli}

Departments of Anatomy and Neurobiology, Pharmacology and Biological Chemistry, University of California, Irvine, CA 92697 , USA

\section{Introduction}

Sphingolipids are a class of bioactive lipid molecules characterized by a high degree of structural and functional diversity. They have been implicated in a variety of biological processes, including senescence [1], inflammation [2], and apoptosis $[3,4]$. Furthermore, the plasma levels of these compounds may be altered in age-related disorders such as Alzheimer's disease [5, 6] and mild cognitive impairment [7]. Sphingolipids also play important roles in cancer [8], diabetes [9], skin disease [10], atherosclerosis [11], and the pathogenesis of obesity [12]. Given their varied and important functions in health and disease, it is essential to be able to measure the concentrations of these biomolecules in plasma and tissues with sensitive, accurate, and reliable methods. Moreover, because of the tight metabolic interconnections among different members of the sphingolipid family, it is important to perform such measurements simultaneously on multiple rather than individual components of the family. Key products and intermediates in sphingolipid metabolism are illustrated in Fig. 1. In the de novo synthesis pathway, sphinganine is acylated to form dihydroceramides, which are converted into ceramides by the action of a desaturase. Ceramides and their corresponding sphingomyelins are metabolically connected through sphingomyelin synthase, which catalyzes the transfer of a polar choline head group onto the relatively apolar structure of ceramides. The reverse reaction, catalyzed by sphingomyelinase, represents the so-called "salvage pathway" for ceramide formation [13]. Glucosyl- and galactosyltransferases converts ceramides into glucosylceramides and galactosylceramides, respectively. Sphingosine and sphinganine (SPH d18:1 and SPH d18:0) are phosphorylated by the action of various kinases. The signaling functions of sphingosine-1-phosphate (S1P d18:1), in particular, are currently under extensive investigation since the discovery in 1998 that S1P d18:1 is an extracellular ligand for S1PR1 


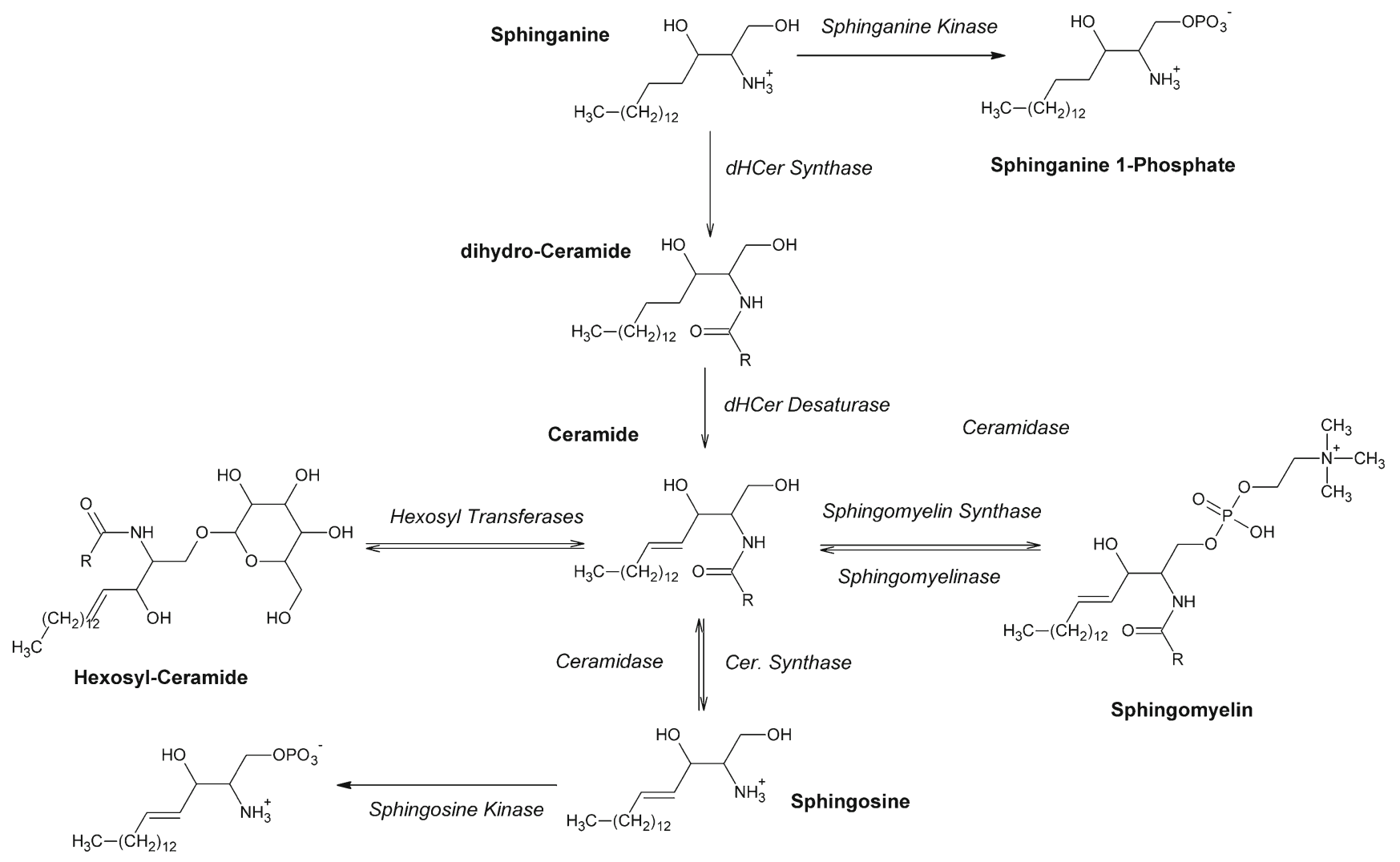

Sphingosine 1-Phosphate

Fig. 1 Key products and intermediates in human sphingolipidome

receptors [14] involved in cancer development and immune responses. An important role in kidney protection after hepatic ischemia has also been recently proved for sphinganine-1phosphate (S1P d18:0) [15]. These evidences underscore the need for analytical tools, allowing the simultaneous detection of SPH d18:1, SPH d18:0, and their corresponding phosphate analogues, along with the other major members of this lipid class like ceramides and sphingomyelins. A variety of technical approaches have been reported for the quantification of sphingolipids in biological matrices. These include thin-layer chromatography (TLC) [16], high-performance liquid chromatography (HPLC) [17], enzymatic hydrolysis followed by HPLC [18], and immunological assays [19]. In recent years, LC-MS/MS-based methods largely imposed themselves over other approaches [20-24]. However, most of these LC-MS/ MS methods suffer from substantial disadvantages, which include low throughput [22], lack of full validation [10, 24], or incomplete analytical coverage (e.g., sphingomyelins) [25]. Substantial advances in lipidome investigation have also been made with shotgun-like workflows, either using combined precursor or neutral loss scan modes [26] or in scan mode using high-resolution instruments [27], but triple-quadrupole-based, targeted approaches will still represent the reference tools for clinical applications in the near future. In this field, indeed, robustness, high-throughput, and easiness of the procedures are the most important benchmarks. The simultaneous analysis of the sphingolipid metabolism (Fig. 1) is extremely challenging due to the structural heterogeneity of the sphingolipidome and the broad polarity span of its members. Differences in polarity, for example, make it difficult to group together S1P d18:1 $(\log \mathrm{P}=3.43)$ and Cer d18:1/24:0 $(\log \mathrm{P}=$ $14.42)$ in the same sample preparation and the same chromatographic run. Even more challenging is to achieve their efficient separation in a short analysis time, ensuring at the same time robustness, reproducibility, and sensitivity. Several new sample preparation methods have also been established like dried blood spot extraction [28] and solid-phase extraction followed by in situ derivatization [29]. Despite these advances, the well-known Bligh-Dyer [30] total lipid extraction still remains very popular, thanks to its efficacy and ease. Here, we describe an LC-MS/MS method for the simultaneous identification and quantification of 25 key members of the sphingolipidome in human plasma, including S1P d18:1 and S1P d18:0. To our knowledge, this is the first reported LC-MS/MS method suitable for the simultaneous evaluation of the large majority of sphingolipid class representatives, and most importantly, this is the first report of an effective incorporation of sphingosine and sphinganine phosphates in a reversed-phase method focused on ceramides and sphingomyelins. The method is sensitive and robust, and it 
has been validated following FDA guidelines, testing for recovery, process efficiency, and matrix effects from human plasma.

\section{Materials and methods}

\section{Materials}

Sphingolipid standards were purchased from Avanti Polar Lipids (Alabaster, Alabama USA). Solvents and chemicals were from Sigma-Aldrich (Milan, Italy). UPLC/MS and MS/ MS systems and columns were from Waters (Milford, USA).

Human plasma samples

Healthy male and female subjects were enrolled in the MCI/ AD Italian prevention project $[31,32]$ aimed at studying cognitive and neuropsychiatric symptoms and disorders in patients with MCI and AD at the IRCCS Santa Lucia Foundation memory clinic in Rome, Italy. The nature and purpose of the study were presented to patients and caregivers and controls, and written informed consent was obtained. The study was approved by the Ethical Committee of the Santa Lucia Foundation. Human plasma samples were collected from healthy male and female volunteers of all ages. Blood samples were taken by venipuncture in the morning after an overnight fast. Blood was collected into $10 \mathrm{ml}$ tubes containing spray-coated K2EDTA (Vacutainer, Becton Dickinson, Italy). Plasma was then prepared by centrifugation of blood at $400 \times \mathrm{g}$ for $15 \mathrm{~min}$, then stored at $-80{ }^{\circ} \mathrm{C}$ before analysis. Blood drawing and sample preparation of human plasma samples were carried out applying the best safety precautions. Plasma samples from all subjects were then pooled together and used as naïve matrix for the present study.

\section{Stock solution preparation}

Stock solutions were prepared in methanol and chloroform mixture (1:1). An internal standard (IS) solution (200 nM Cer d18:1/17:0, $200 \mathrm{nM}$ PC 23:0/23:0, $200 \mathrm{nM}$ GlcCer d18:1/12:0, $250 \mathrm{nM}$ SPH d17:1, $250 \mathrm{nM}$ SPH d17:0, $250 \mathrm{nM} \mathrm{S1P}$ d17:1, and $250 \mathrm{nM} \mathrm{S1P} \mathrm{d17:0)} \mathrm{was} \mathrm{prepared}$ by spiking the corresponding standards in the extraction solvent methanol/chloroform (2:1) added with trifluoroacetic acid (TFA) to a final $0.1 \%(v / v)$ concentration.

Sample preparation

Samples were extracted using a Bligh and Dyer method for lipid extraction [30]. Calibration curve (CC), quality control (QC), or test samples $(50 \mu \mathrm{L})$ were transferred to glass vials. Liquidliquid extraction (LLE) was carried out using a 1:2 by volume chloroform/methanol mixture $(2 \mathrm{ml})$ added with TFA (final $0.1 \% v / v)$ and spiked with the IS as described above. After mixing for $30 \mathrm{~s}$ with a Vortex ${ }^{\circledR}$, chloroform $(0.5 \mathrm{~mL})$ and water $(0.5 \mathrm{~mL})$ were sequentially added, thoroughly mixing after each addition. The samples were then centrifuged for $15 \mathrm{~min}$ at $3500 \times g$ at room temperature. At the end of the process, the aqueous (upper) and organic (lower) phases were separated by a protein precipitate floating at the interface. The organic phase was then transferred to glass vials. To increase the overall recovery, the aqueous fraction was extracted again with chloroform $(1 \mathrm{~mL})$. The two resulting organic phases were pooled, dried under a stream of $\mathrm{N}_{2}$, and the residues were redissolved in methanol/chloroform ( $9: 1$, by volume; $0.1 \mathrm{~mL})$. After mixing (30 s) and centrifugation ( $10 \mathrm{~min}$ at $5000 \times g$, room temperature), the samples were transferred to glass vials for analyses.

\section{LC-MS/MS analyses}

LC-MS/MS analyses of the samples were carried out on an Acquity UPLC system coupled with a Xevo TQ-MS triplequadrupole mass spectrometer. Chromatographic separation was achieved using a BEH C18 column $(2.1 \times 50 \mathrm{~mm}, 1.7 \mathrm{mi}-$ cron particle size) eluted at a flow rate of $0.4 \mathrm{~mL} / \mathrm{min}$. Instruments and column were from Waters Inc. Milford, MA, USA. The mobile phase consisted of $0.1 \%$ formic acid in acetonitrile/water $(20: 80 \mathrm{v} / \mathrm{v})$ as solvent $\mathrm{A}$ and $0.1 \%$ formic acid in acetonitrile/2-propanol $(20: 80 v / v)$ as solvent B. A step gradient program was developed for the best separation of all metabolites: $0.0-1.0 \min 30 \% \mathrm{~B}, 1.0-2.5 \min 30$ to $70 \% \mathrm{~B}, 2.5$ $4.0 \mathrm{~min} 70$ to $80 \% \mathrm{~B}, 4.0-5.0 \mathrm{~min} 80 \% \mathrm{~B}, 5.0-6.5 \mathrm{~min} 80$ to $90 \% \mathrm{~B}$, and $6.6-7.5 \mathrm{~min} 100 \% \mathrm{~B}$. The column was then reconditioned to $30 \% \mathrm{~B}$ for $1.4 \mathrm{~min}$. The total run time for analysis was $9 \mathrm{~min}$, and the injection volume was $3 \mu \mathrm{L}$. The mass spectrometer was operated in the positive ESI mode, and analytes were quantified by multiple reaction monitoring (MRM). The capillary voltage was set at $3 \mathrm{kV}$. The cone voltage was set at $25 \mathrm{~V}$ for all transitions, except for some SM. The complete panel of source parameters and MRM transitions are reported in the datasheet in the Electronic Supplementary Material (ESM). The source temperature was set to $120{ }^{\circ} \mathrm{C}$. Desolvation gas and cone gas $\left(\mathrm{N}_{2}\right)$ flow were set to 800 and $20 \mathrm{l} / \mathrm{h}$, respectively. Desolvation temperature was set to $600^{\circ} \mathrm{C}$. Data were acquired by MassLynx software and quantified by TargetLynx software. Calibration curves were constructed by plotting the analyte to IS peak areas ratio versus the corresponding analyte concentration using weighted $\left(1 / x^{2}\right)$ least square regression analysis, as recommended by $\mathrm{Gu}$ and colleagues [33].

Standard curves

As analytes are present endogenously in plasma, $5 \%$ bovine serum albumin (BSA) in saline solution was used as surrogate matrix [34] for the first part of the validation process (see the 
"Results and discussion" for additional information). Calibration standards were prepared by spiking the analytes in the surrogate matrix. Eight-point calibration curves (1 to $1000 \mathrm{nM}$ ) were prepared by serial dilution into saline solution containing $5 \%$ BSA.

\section{Quality control samples}

Quality control samples were prepared at three different levels, as low QC (LQC), medium QC (MQC), and high QC (HQC), using the same procedure described for the standard curve to final concentrations of $19.5,260$, and $650 \mathrm{nM}$, respectively.

Linearity, precision, and accuracy

Method linearity was tested using the eight-point calibration curve described above. The overall method performance was assessed by evaluating the accuracy and precision of backcalculated concentrations of standards and evaluating the slope, intercept, and coefficient of determination of the 1/(concentration $)^{2}$ weighted regression line. Following FDA guidelines in this matter, acceptance criteria for the calibration curve were set to $\pm 15 \%$ of the nominal concentration and six out of eight calibration points had to meet the acceptance criteria. Precision and accuracy were determined by assessing the performance of quality control samples (LQC $=19.5 \mathrm{nM}, \mathrm{MQC}=$ $260 \mathrm{nM}$, and $\mathrm{HQC}=650 \mathrm{nM}$ ). All the QC samples were run in triplicate. Accuracy was evaluated by calculating the percent deviation (\% dev.) from nominal concentration. Precision was determined by calculating the coefficient of variation (\% CV) of replicates within each batch. Acceptance criteria for precision and accuracy were defined as $\leq 15 \%$ [35].

\section{LLOQ}

The lower limit of quantification for our method was set to $1 \mathrm{nM}$ for all analytes. At this value, all species showed a $\mathrm{S} / \mathrm{N}$ value above 10. Six replicate 1-nM calibrators spiked in $5 \%$ BSA were extracted and analyzed. The obtained $\% \mathrm{CV}$ values for each analyte are reported in the ESM (supplementary datasheet).

Sample recovery and matrix effects

Recovery and matrix effects were evaluated using the method outlined by Matuszewski et al. [36]. Three sets of samples were prepared spiking the standards at three different concentrations. Set 1 consisted of neat samples (standards spiked in reconstitution solution). Set 2 consisted of post-extraction spiked samples (blank matrix was extracted and then spiked with standards). Set 3 consisted of normal extracted samples (standards were spiked in blank matrix and then extracted).
Matrix effect (ME) was calculated as (set $2 /$ set 1$) \times 100$. Recovery (RE) was calculated as (set 3/set 2) $\times 100$. Human plasma matrix effect was evaluated using the post-column infusion method: A mixture of analytes diluted in 9:1 $\mathrm{MeOH} / \mathrm{CHCl}_{3}$ to a final $10 \mu \mathrm{M}$ concentration was infused post-column in the LC-MS/MS system using a tee union. Repeated injections of extracted human plasma samples were performed in the system with the aim to investigate significant decreases or increases in the analyte MRM ion currents.

\section{Plasma recovery}

Analyte recovery from plasma was calculated using the same criteria described above: A mixture of standards was added at different concentrations in plasma (from $50 \mathrm{nM}$ to $15 \mu \mathrm{M}$ ) prior or after the extraction (pre- and post-extraction spiking). The recovery was calculated as the ratio between the analyte peak area pre-spiked in plasma and the analyte peak area in post-extraction spiked samples (see "Results and discussion" for detailed information).

Autosampler and plasma stability and dilution integrity

Plasma stability was assessed by spiking odd-chain standards in human plasma and keeping the sample under different temperature and timing conditions: short-term conditions (4 and $25^{\circ} \mathrm{C}$ for $\left.6 \mathrm{~h}\right)$ and long-term storage conditions $\left(-20^{\circ} \mathrm{C}\right.$ for 9 days). Sample stability in the instrument autosampler was also evaluated by re-analyzing extracted samples kept under the autosampler condition $\left(18 \mathrm{~h}\right.$ at $\left.4{ }^{\circ} \mathrm{C}\right)$. All the stability studies were conducted in triplicate at $500 \mathrm{nM}$ concentration. Method integrity to dilutions was also evaluated by spiking odd-chain standards in human plasma to a final $4000 \mathrm{nM}$ concentration. Samples were then diluted 20-fold in blank human plasma, extracted, and analyzed.

\section{Results and discussion}

\section{Chromatographic separation}

We tested multiple combinations of stationary phases and elution conditions and eventually selected a gradient elution with an initial solvent system of low organic content, which was required to retain on the column polar compounds such as SPH d18:1 and SPH d18:0, followed by a rapid progressive increase in solvent strength, which was needed to elute from the column apolar compounds such as ceramides and dihydroceramides. The best compromise between selectivity and speed of separation was obtained using a C18 stationary phase and a high content of 2-propanol (80\%) in solvent B coupled to $20 \% \mathrm{ACN}$ in solvent $\mathrm{A}$ to reduce back pressure. Using these conditions, an adequate separation of 25 target 
analytes was achieved in a single 9-min-long LC-MS run (Fig. 2). In the interest of clarity, the analyte traces shown in Fig. 2 are split in four panels (A: separation of ceramides; B: dihydroceramides; C: sphingomyelins, SPH d18:1, SPH d18:0, S1P d18:1, and S1P d18:0; D: glucosylceramides).

MS parameter optimization and choice of internal standards

To define MS parameters for optimum sensitivity, authentic standards were infused separately into the mass spectrometer. The most intense MRM transition was selected to quantify each analyte (see ESM supplementary datasheet). A total of $32 \mathrm{MRM}$ transitions were included in the method. Although it is very well known that deuterated standard represents the best choice as internal standards for LC-MS/MS analysis of endogenous molecules since a very limited number of deuterated sphingolipids are commercially available, and due to the very high cost of the custom synthesis of a deuterated standard for each of the 25 sphingolipids we are tracking, we were forced to set up and validate our method using odd-chain lipids as internal standards for analytes of similar structure but with even-numbered carbon chains. The use of odd-chain standards is a very common approach for sphingolipid analysis [37], and odd-chain Cer d18:1/17:0 has already been successfully used by Haus and colleagues to determine ceramide content in plasma [9]. Since no odd-chain glucosylceramides are commercially available, we used instead the short-chain GlcCer $\mathrm{d} 18: 1 / 12: 0$, which was not detectable in plasma. It is important to point out that our RP method cannot distinguish between gluco- and galactosylceramides that are fully coeluting. We then chose to use glucosylceramides for the calibration curve and GlcCer d18:1/12:0 as internal standard for both gluco- and galacto- derivatives, assuming an identical instrumental response in ESI conditions, as recently confirmed by Shaner and colleagues [38]. For the same reason (full coelution and identical instrumental response) and to limit additional costs, we only used glucosylceramides in the calibration curve. Our method is then intended to evaluate the total hexosylceramides content in plasma. Notably, SM d18:1/ 17:0 did not provide a suitable internal standard for endogenous sphingomyelin species because its MRM transition $(\mathrm{m} / \mathrm{z}$ 718->184) was found to generate an interfering peak (Fig. 3). Having excluded other options, we then concluded that SM $\mathrm{d} 18: 1 / 17: 0$ is endogenously present in plasma and we tested other exogenous lipid molecules potentially suitable as internal standards for sphingomyelins (see ESM Fig. S1). We tested human plasma for the MRM transitions of PC 16:0/16:0 (used by Byrdwell and Perry [39]), SM d18:1/12:0, PC 9:0/ 9:0, PC 15:0/15:0, and PC 17:0/17:0 (panels A to E, respectively). All these transitions generated huge ion currents (>5000-10,000 ion counts) at multiple retention times. These peaks correspond to isomeric (even/even) phosphatidylcholines like, for example, (16:0/18:0) for (17:0/17:0). We then discarded all the corresponding molecules as internal standards. The best alternative option would have been an (odd/even) phosphatidylcholine that is not commercially
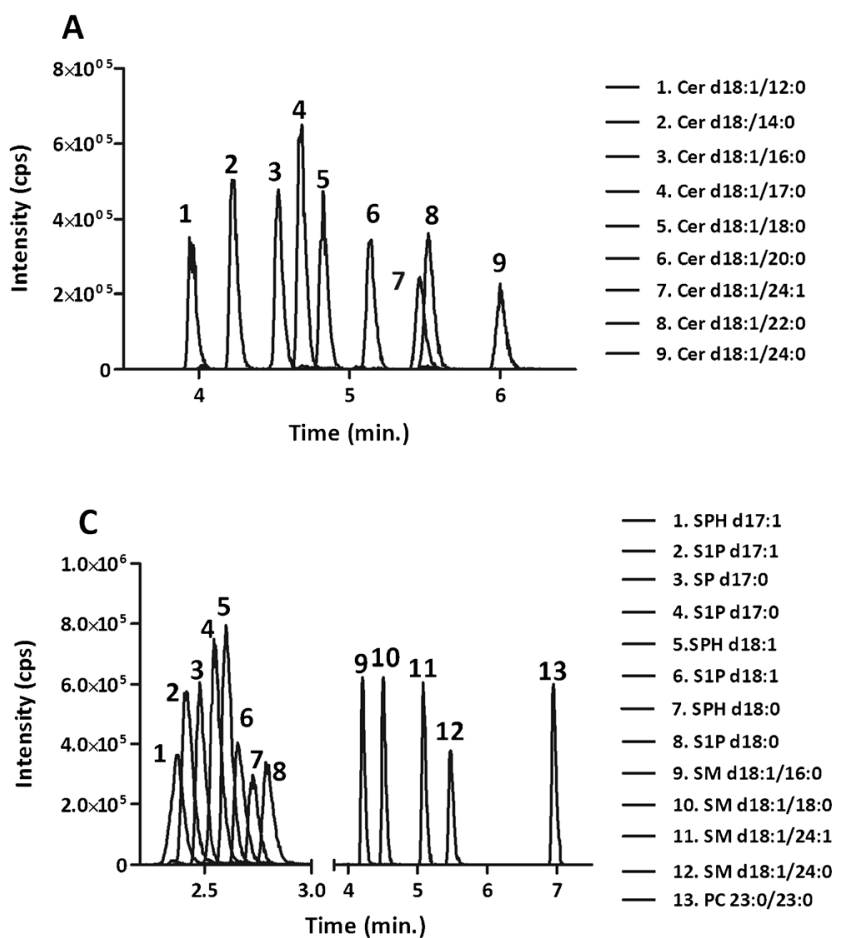
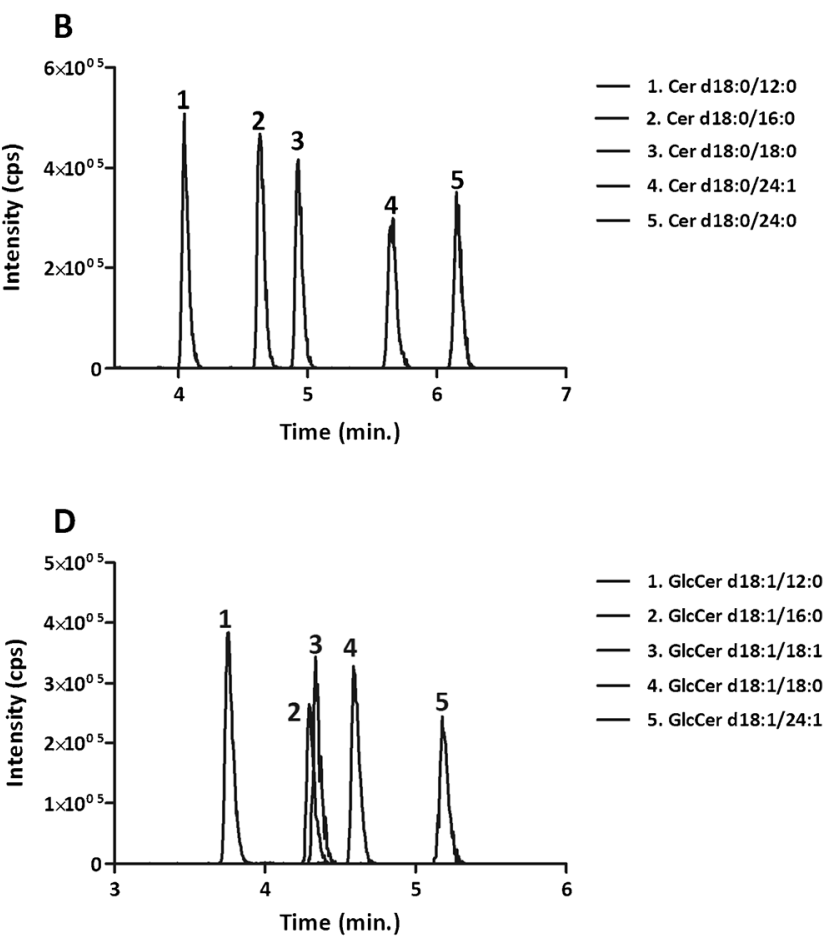

Fig. 2 MRM chromatograms of A ceramides; B dihydroceramides; C sphingomyelins, sphingosines, and sphinganines; and $\mathbf{D}$ glucosylceramides spiked at $1 \mu \mathrm{M}$ in $5 \% \mathrm{BSA}$ 

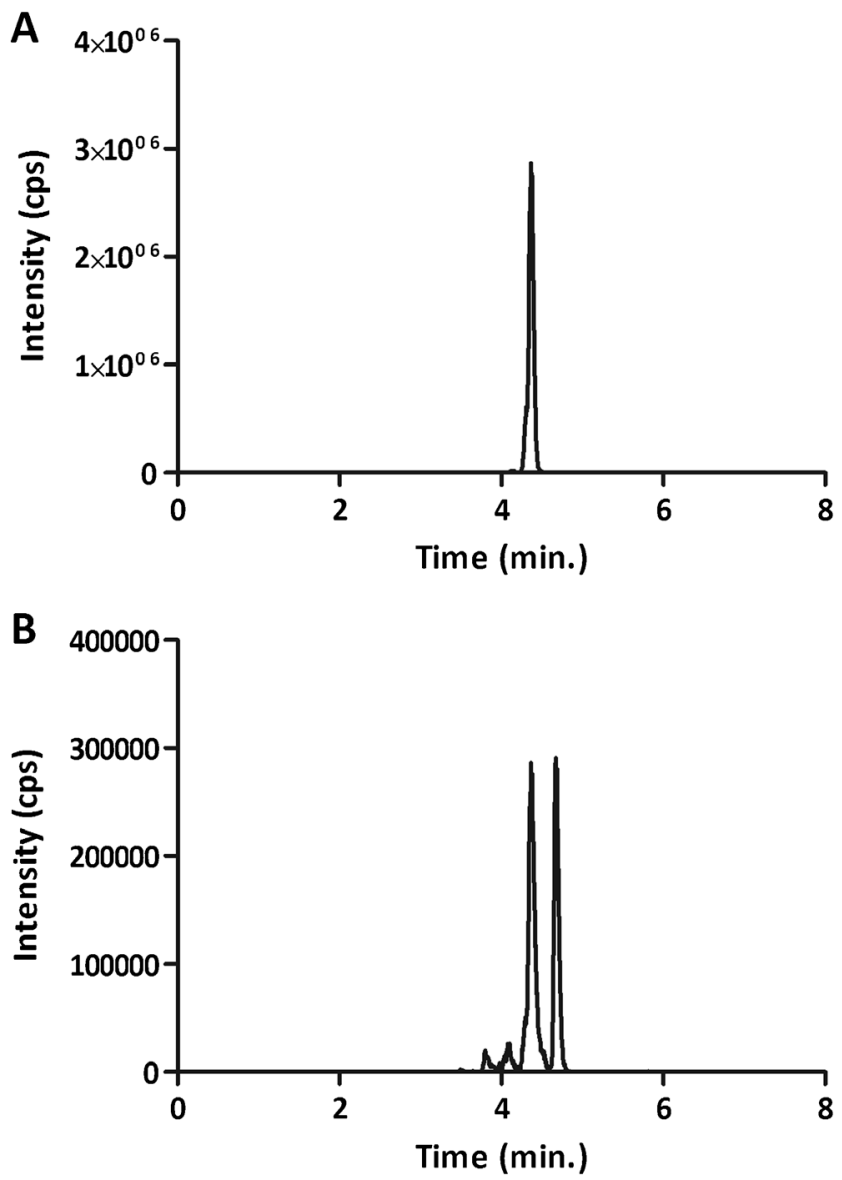

Fig. 3 MRM trace for transition $m / z$ 718->184 from standard SM d18:1/ 17:0 (A) and from extracted naïve plasma (B). An interfering peak in plasma shares the same transition and retention time with standard SM d18:1/17:0

available. We then opted for PC 23:0/23:0 because the corresponding MRM transition from human plasma (ESM Fig. S1, panel F, black trace) generates the lowest ion current $(<1500$ ion counts) and does not interfere with the detection and quantification of authentic PC 23:0/23:0 (red trace).

A detailed summary of the MRM transitions and source parameters for all analytes and internal standards is reported in the ESM (supplementary datasheet). Since we are fully aware that the lack of a coeluting internal standard might represent a major problem in LC-MS/MS, we did our best to evaluate possible matrix effects using the post-column infusion method [40]. The results of this experiment are available for download as ESM. Most analytes did not show any relevant matrix effect when extracted human plasma samples were injected. Five analytes showed non-negligible matrix effects with three out of four glucosylceramides among them $(16: 0,18: 0$, and 18:1). It is thus important to point out that, assuming identical matrix effects for galactosylceramides, the evaluation of hexosylceramides content in plasma might be affected, in the absence of commercially available deuterated standards. As expected, injections of extracted plasma caused a positive increase in the post-column infusion trace of those metabolites that are endogenously present in plasma at micromolar concentrations (sphingomyelins mostly). We also investigated the possibility of endogenous plasma phosphatidylcholines coeluting with the four sphingomyelins quantified by our method and having similar $m / z$ values. For the same reasons indicated above (common $184 \mathrm{~m} / \mathrm{z}$ fragment), this would result in interferences and inaccuracy in sphingomyelins quantification. We addressed this issue by means of highresolution MS, a brute formula calculation software and LipidMaps database search, and we showed that, at the retention time of the four sphingomyelins targeted by our method, no phosphatidylcholines having a $\mathrm{m} / \mathrm{z}$ value close to that selected for fragmentation $( \pm 0.5 \mathrm{~m} / \mathrm{z}$ units $)$ is coeluting. The results of this experiment, along with a detailed description of the experiment, are available for download as ESM.

\section{Sample preparation}

Sphingolipids are naturally present in plasma, where they can reach relatively high concentrations $(10-20 \mu \mathrm{M}$ for certain species). This hindered the use of plasma as blank analytical matrix and interfered with our ability to validate the method. Since depletion strategies (for example, using charcoal [41, 42]) did not perform well in our hands, we switched to a surrogate plasma-like matrix constituted of a saline solution containing diluted, lipid-free, bovine serum albumin (BSA, $5 \mathrm{~g} / 0.1 \mathrm{~L}$ ), which has been widely used in the past to mimic plasma in bioanalysis [43-45]. We fully validated the method using this artificial matrix. Linearity, accuracy, precision, matrix effect, and recovery were evaluated for each analyte. Full validation data are available for download as ESM. Next, we determined linearity and recovery in human plasma samples. We first used odd-numbered chain internal standards and GlcCer(d18:1/12:0) to test process efficiency: Different combinations of analytes to extraction solution (ES) volume ratios were examined. We found that while a 1:6 ratio was adequate for $5 \%$ BSA, a 1:40 ratio was required to ensure a good recovery from plasma. We then focused on S1P(d18:1) and S1P(d18:0), where the hydrophilic/hydrophobic dichotomy of sphingolipids is particularly relevant. At neutral $\mathrm{pH}$, these molecules have a positively charged amino group and a negatively charged phosphate group, along with a 14 carbon long aliphatic chain. Without changes in the $\mathrm{pH}$, the extraction efficiency of S1P(d18:1) and S1P(d18:0) from plasma is rather low (approximately $20 \%$ for both) with a large fraction $(>75 \%)$ of analyte that remains in the upper aqueous phase. We then added TFA to the extraction solvent $(0.1 \%$ in volume) to force the protonation of both the phosphate group and the amino group, reducing the span in the protonation states. The trifluoroacetate anion also served as ion-pairing agent. TFA was chosen due to its volatility. This expedient increased the recovery of S1P(d18:1) and S1P(d18:0) to more than $80 \%$ 
and had no significant effect on the recovery of other species. With this information in hand, to obtain a more complete picture of the extraction efficiencies from human plasma for all analytes, we prepared a set of human plasma samples spiked with higher-than-background concentrations of standards (from $50 \mathrm{nM}$ to $15 \mu \mathrm{M}$ ). Plasma samples were also extracted, analyzed, and considered as background level. We then plotted the sphingolipids concentration against the total amount of standards added. The amount of sphingolipids naturally present in the naïve samples was subtracted from each point of the graph. Results are reported in Fig. 4. As shown in the graphs, the method yields linear recoveries when applied to human plasma samples. Furthermore, in order to calculate the plasma recovery values for individual analytes, each point was prepared using both pre-extraction and post-extraction spiking for each analyte. The recovery was then calculated from the ratio between pre-extraction and post-extraction spiking. Table 1 shows the averaged recovery values from surrogate matrix and human plasma for each sphingolipid class. The full data panel, with data on individual species, may be found in the ESM (Supplementary Datasheet).

Linearity, precision, accuracy, carryover, stability, and dilution integrity

Our method showed a very good linearity over a $10^{3}$-fold range (1-1000 nM, eight calibration points) with an $r^{2}$ value
Table 1 Recovery values by sphingolipid class (mean \pm standard deviation) from $5 \%$ BSA and from human plasma samples

\begin{tabular}{lll}
\hline Sphingolipids & BSA 5 \% & Human plasma \\
\hline Ceramides & $89.66 \pm 2.40$ & $95.74 \pm 7.21$ \\
Dihydroceramides & $91.53 \pm 1.70$ & $96.89 \pm 5.03$ \\
Sphingomyelins & $82.97 \pm 0.79$ & $97.82 \pm 1.36$ \\
Glucosylceramides & $89.93 \pm 2.54$ & $100.93 \pm 7.15$ \\
SPH(d18:1) & $116.11 \pm 2.94$ & $71.93 \pm 4.19$ \\
SPH(d18:0) & $116.86 \pm 1.16$ & $93.69 \pm 4.90$ \\
S1P(d18:1) & $76.93 \pm 2.45$ & $105.01 \pm 14.08$ \\
S1P(d18:0) & $75.89 \pm 0.71$ & $86.64 \pm 4.21$ \\
\hline
\end{tabular}

of $0.9895-0.9973$ using $5 \%$ BSA as matrix and $0.9947-$ 1.0000 using human plasma as matrix. A signal-to-noise ratio $(\mathrm{S} / \mathrm{N})$ of $\geq 10$ was considered as lowest limit of quantification to determine method sensitivity, although for most analytes the $\mathrm{S} / \mathrm{N}$ at $1 \mathrm{nM}$ was higher than 10 . For this reason, lower limit of quantification (LLOQ) from 5\% BSA was set to $1 \mathrm{nM}$. At this concentration, all analytes showed a $\% \mathrm{CV}$ below $20 \%$ (see ESM, supplementary datasheet). Precision and accuracy were also determined in $5 \% \mathrm{BSA}$, assessing the performance of three quality control samples (LQC $=19.5 \mathrm{nM}, \mathrm{MQC}=$ $260 \mathrm{nM}$, and HQC $=650 \mathrm{nM}$ ). Accuracy was evaluated by calculating the percent deviation from nominal concentrations. Precision was determined by calculating the coefficient

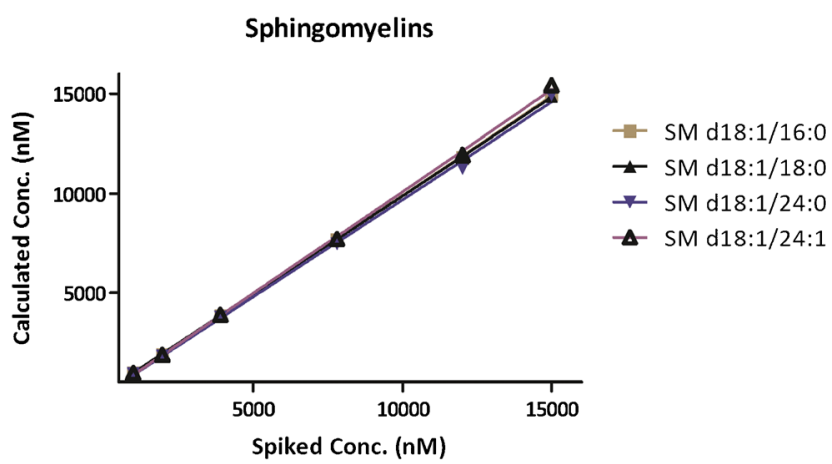

Cer d18:1/24:0 and Cer d18:0/24:0

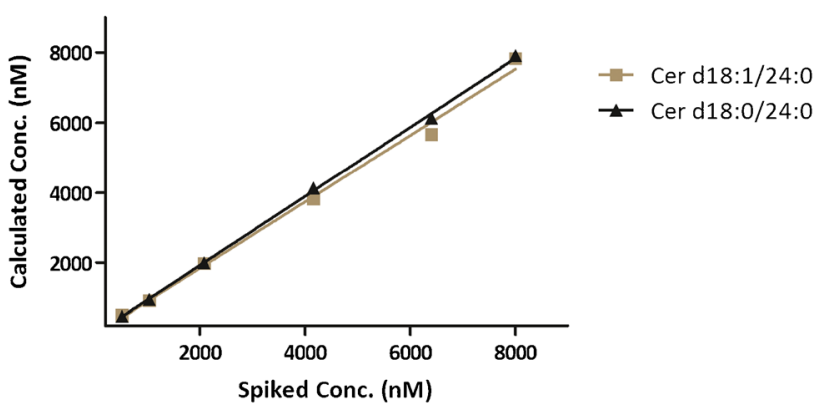

Fig. 4 The plots shows the analyte concentration calculated after extraction and LC-MS/MS analysis versus the amount of authentic standard sphingolipids added to naïve plasma. Pre-spiking naïve plasma levels were considered as background levels and subtracted 
of variation of replicates within each batch. All the QC samples were run in triplicate. Acceptance criteria for precision and accuracy were defined as $\leq 15 \%$ [35]. Intra- and interassay precision and accuracy were also tested by analyzing the same QC samples. The full dataset is reported in the ESM (supplementary datasheet). Furthermore, in order to evaluate carryover, a blank sample was injected immediately after the highest standard. Since some carryover was observed with the usual water/methanol/ACN/2-propanol needle washing system, we used acetonitrile/water $(1: 1)$ containing $10 \%$ acetone as needle wash solvent. With this expedient, no significant carryover was observed for any of the analytes (less than $0.5 \%$ ). Analyte stability evaluation was also included in the experimental layout: Odd-chain standards were spiked in naïve human plasma to a final $500 \mathrm{nM}$ concentration (triplicate samples) and tested for stability in both short-term (4 and $25{ }^{\circ} \mathrm{C}$ for $6 \mathrm{~h}$ ) and long-term conditions ( 9 days at $-20{ }^{\circ} \mathrm{C}$ ). The stability of extracted samples in the instrument autosampler conditions $\left(4^{\circ} \mathrm{C}\right.$ for $\left.6 \mathrm{~h}\right)$ was also evaluated. Analytes are stables in all the abovementioned conditions (our acceptance criteria were set to $\pm 15 \%$ of nominal concentration). Since sphingolipids are present in human plasma at very different concentrations (from low $\mathrm{nM}$ to high $\mu \mathrm{M}$ ), we also tested the dilution integrity of our method in order to be able to confidently quantify samples above the upper limit of the calibration curve. We then spiked odd-chain standards in naïve human plasma at a final $4 \mu \mathrm{M}$ concentration, and we diluted the samples 20 -fold using naïve human plasma. After sample extraction and analysis, analyte concentrations were calculated using the appropriate multiplication factor. Results demonstrate that our method is tolerant to 20 -fold dilution since the calculated concentrations were within the acceptance criteria (precision 0.16-4.82\%, accuracy 83.04-94.25\%). Data on stability and dilution integrity are reported in the ESM (supplementary datasheet). All the sphingolipids that were detected and quantified with our method in naïve human plasma are reported in the supplementary datasheet in the ESM.

\section{Conclusions}

In the present report, we describe a new LC-MS/MS method that allows for the rapid identification and quantification of 25 key sphingolipid species in human plasma, including S1P d18:1 and S1P d18:0. We proved that a $\log P$ span of more than 10 units (from 3.43 of S1P d18:1 to 14.42 of Cer d18:1/ 24:0 can be efficiently explored in a single sample preparation and LC-MS run. While other validated methods for the LCMS/MS quantification of sphingolipids are available in the literature, none of them offers a comparable level of analyte coverage, rapidity, and validation. For example, Bui et al. [25] describe a 5-min separation but do not cover sphingomyelins;
Kasumov and colleagues [22] describe a separation of seven species in $15 \mathrm{~min}$. Other methods, though validated, offer an even more limited analyte coverage: Cer d18:1/22:0 and Cer d18:1/24:0 [23] or seven species by nano-LC/MS [21]. The method we propose here is sensitive, linear, robust, and very rapid ( 9 min per run). Although it suffers from some matrix effect that cannot be fully compensated by the use of oddchain internal standards, we managed to evaluate this effect and control it, achieving very promising results from human plasma. Future implementations of this method with custommade deuterated internal standards will hopefully improve the overall method performance, making it suitable for clinical routine. The huge costs for custom synthesis will surely be compensated by the final outcome on health-care systems since sphingolipids and phosphorylated sphingolipids have been proposed as biomarkers for various pathological conditions. The potential of the method presented here is manifested as it permits a complete and reliable analysis of the full sphingolipid core metabolism in less than $10 \mathrm{~min}$. Furthermore, in addition to the whole sphingolipids panel, the possibility to track S1P d18:1 and S1P d18:0 in plasma could be of great importance for clinical research on cancer and immune system disorders.

Acknowledgments The authors would like to thank Dr. Gianfranco Spalletta (IRCCS Santa Lucia, Rome, Italy) for kindly providing human plasma samples from healthy donors. The financial support of the National Institutes of Health (grants DK073955 and DA012413 to D.P.) is gratefully acknowledged.

Open Access This article is distributed under the terms of the Creative Commons Attribution License which permits any use, distribution, and reproduction in any medium, provided the original author(s) and the source are credited.

\section{References}

1. Webb LM, Arnholt AT, Venable ME (2010) Phospholipase D modulation by ceramide in senescence. Mol Cell Biochem 337(1-2):153158

2. Teichgräber V, Ulrich M, Endlich N, Riethmüller J, Wilker B, De Oliveira-Munding CC, van Heeckeren AM, Barr ML, von Kürthy G, Schmid KW, Weller M, Tümmler B, Lang F, Grassme H, Döring G, Gulbins E (2008) Ceramide accumulation mediates inflammation, cell death and infection susceptibility in cystic fibrosis. Nat Med 14(4):382-391

3. Posse de Chaves EI (2006) Sphingolipids in apoptosis, survival and regeneration in the nervous system. Biochim Biophys Acta 1758(12): 1995-2015

4. Young MM, Kester M, Wang HG (2013) Sphingolipids: regulators of crosstalk between apoptosis and autophagy. J Lipid Res 54(1):5-19

5. He X, Huang Y, Li B, Gong CX, Schuchman EH (2010) Deregulation of sphingolipid metabolism in Alzheimer's disease. Neurobiol Aging 31(3):398-408 
6. Mielke MM, Haughey NJ, Bandaru VV, Weinberg DD, Darby E, Zaidi N, Pavlik V, Doody RS, Lyketsos CG (2011) Plasma sphingomyelins are associated with cognitive progression in Alzheimer's disease. J Alzheimers Dis 27(2):259-269

7. Mielke MM, Haughey NJ, Ratnam Bandaru VV, Schech S, Carrick R, Carlson MC, Mori S, Miller MI, Ceritoglu C, Brown T, Albert M, Lyketsos CG (2010) Plasma ceramides are altered in mild cognitive impairment and predict cognitive decline and hippocampal volume loss. Alzheimers Dement 6(5):378-385

8. Bieberich E (2008) Ceramide signaling in cancer and stem cells. Futur Lipidol 3(3):273-300

9. Haus JM, Kashyap SR, Kasumov T, Zhang R, Kelly KR, Defronzo RA, Kirwan JP (2009) Plasma ceramides are elevated in obese subjects with type 2 diabetes and correlate with the severity of insulin resistance. Diabetes 58(2):337-343

10. Farwanah H, Pierstorff B, Schmelzer CE, Raith K, Neubert RH, Kolter T, Sandhoff K (2007) Separation and mass spectrometric characterization of covalently bound skin ceramides using LC/APCI-MS and Nano-ESI-MS/MS. J Chromatogr B Anal Technol Biomed Life Sci 852(1-2):562-570

11. Bismuth J, Lin P, Yao Q, Chen C (2008) Ceramide: a common pathway for atherosclerosis? Atherosclerosis 196(2):497-504

12. Samad F, Hester KD, Yang G, Hannun YA, Bielawski J (2006) Altered adipose and plasma sphingolipid metabolism in obesity: a potential mechanism for cardiovascular and metabolic risk. Diabetes 55(9):2579-2587

13. Kitatani K, Idkowiak-Baldys J, Hannun YA (2008) The sphingolipid salvage pathway in ceramide metabolism and signaling. Cell Signal 20(6):1010-1018

14. Hanson MA, Roth CB, Jo E, Griffith MT, Scott FL, Reinhart G, Desale H, Clemons B, Cahalan SM, Schuerer SC, Sanna MG, Han GW, Kuhn P, Rosen H, Stevens RC (2012) Crystal structure of a lipid G protein-coupled receptor. Science 335(6070):851-855

15. Park SW, Kim M, Chen SW, Brown KM, D'Agati VD, Lee HT (2010) Sphinganine-1-phosphate protects kidney and liver after hepatic ischemia and reperfusion in mice through S1P1 receptor activation. Lab Investig 90(8):1209-1224

16. Bruce CR, Thrush AB, Mertz VA, Bezaire V, Chabowski A, Heigenhauser GJ, Dyck DJ (2006) Endurance training in obese humans improves glucose tolerance and mitochondrial fatty acid oxidation and alters muscle lipid content. Am J Physiol Endocrinol Metab 291(1):E99-E107

17. Yano M, Kishida E, Muneyuki Y, Masuzawa Y (1998) Quantitative analysis of ceramide molecular species by high performance liquid chromatography. J Lipid Res 39(10):2091-2098

18. Lee S, Lee YS, Choi KM, Yoo KS, Sin DM, Kim W, Lee YM, Hong JT, Yun YP, Yoo HS (2012) Quantitative analysis of sphingomyelin by high-performance liquid chromatography after enzymatic hydrolysis. Evid Based Complement Alternat Med 2012:396218

19. Cowart LA, Szulc Z, Bielawska A, Hannun YA (2002) Structural determinants of sphingolipid recognition by commercially available anti-ceramide antibodies. J Lipid Res 43(12):2042-2048

20. Liou YB, Sheu MT, Liu DZ, Lin SY, Ho HO (2010) Quantitation of ceramides in nude mouse skin by normal-phase liquid chromatography and atmospheric pressure chemical ionization mass spectrometry. Anal Biochem 401(1):107-113

21. Thomas D, Eberle M, Schiffmann S, Zhang DD, Geisslinger G, Ferreiros N (2013) Nano-LC-MS/MS for the quantitation of ceramides in mice cerebrospinal fluid using minimal sample volume. Talanta 116:912-918

22. Kasumov T, Huang H, Chung YM, Zhang R, McCullough AJ, Kirwan JP (2010) Quantification of ceramide species in biological samples by liquid chromatography electrospray ionization tandem mass spectrometry. Anal Biochem 401(1):154-161

23. Jiang H, Hsu FF, Farmer MS, Peterson LR, Schaffer JE, Ory DS, Jiang X (2013) Development and validation of LC-MS/MS method for determination of very long acyl chain (C22:0 and C24:0) ceramides in human plasma. Anal Bioanal Chem 405(23):73577365

24. Farwanah H, Kolter T, Sandhoff K (2011) Mass spectrometric analysis of neutral sphingolipids: methods, applications, and limitations. Biochim Biophys Acta 1811(11):854-860

25. Bui HH, Leohr JK, Kuo MS (2012) Analysis of sphingolipids in extracted human plasma using liquid chromatography electrospray ionization tandem mass spectrometry. Anal Biochem 423(2):187194

26. Eisinger K, Krautbauer S, Hebel T, Schmitz G, Aslanidis C, Liebisch G, Buechler C (2014) Lipidomic analysis of the liver from high-fat diet induced obese mice identifies changes in multiple lipid classes. Exp Mol Pathol 97(1):37-43

27. Schuhmann K, Almeida R, Baumert M, Herzog R, Bornstein SR, Shevchenko A (2012) Shotgun lipidomics on a LTQ Orbitrap mass spectrometer by successive switching between acquisition polarity modes. J Mass Spectrom 47(1):96-104

28. Legnini E, Orsini JJ, Muhl A, Johnson B, Dajnoki A, Bodamer OA (2012) Analysis of acid sphingomyelinase activity in dried blood spots using tandem mass spectrometry. Ann Lab Med 32(5):319-323

29. Sanchez BA, Capote FP, Luque de Castro MD (2011) Targeted analysis of sphingoid precursors in human biofluids by solid-phase extraction with in situ derivatization prior to mu-LC-LIF determination. Anal Bioanal Chem 400(3):757-765

30. Bligh EG, Dyer WJ (1959) A rapid method of total lipid extraction and purification. Can J Biochem Physiol 37(8):911-917

31. Palmer K, Di Iulio F, Varsi AE, Gianni W, Sancesario G, Caltagirone C, Spalletta G (2010) Neuropsychiatric predictors of progression from amnestic-mild cognitive impairment to Alzheimer's disease: the role of depression and apathy. J Alzheimers Dis 20(1):175-183

32. Di Iulio F, Palmer K, Blundo C, Casini AR, Gianni W, Caltagirone C, Spalletta G (2010) Occurrence of neuropsychiatric symptoms and psychiatric disorders in mild Alzheimer's disease and mild cognitive impairment subtypes. Int Psychogeriatr 22(4):629-640

33. Gu H, Liu G, Wang J, Aubry AF, Arnold ME (2014) Selecting the correct weighting factors for linear and quadratic calibration curves with least-squares regression algorithm in bioanalytical LC-MS/MS assays and impacts of using incorrect weighting factors on curve stability, data quality, and assay performance. Anal Chem 86(18): 8959-8966

34. van de Merbel NC (2008) Quantitative determination of endogenous compounds in biological samples using chromatographic techniques. Trends Anal Chem 27(10):924-933

35. Booth B, Arnold ME, DeSilva B, Amaravadi L, Dudal S, Fluhler E, Gorovits B, Haidar SH, Kadavil J, Lowes S, Nicholson R, Rock M, Skelly M, Stevenson L, Subramaniam S, Weiner R, Woolf E. (2014) Workshop report: crystal city V-Quantitative bioanalytical method validation and implementation: the 2013 revised FDA guidance. AAPS journal. doi:10.1208/s12248-014-9696-2

36. Matuszewski BK, Constanzer ML, Chavez-Eng CM (2003) Strategies for the assessment of matrix effect in quantitative bioanalytical methods based on HPLC-MS/MS. Anal Chem 75(13):3019-3030

37. Lan T, Bi H, Liu W, Xie X, Xu S, Huang H (2011) Simultaneous determination of sphingosine and sphingosine 1-phosphate in biological samples by liquid chromatography-tandem mass spectrometry. J Chromatogr B Anal Technol Biomed Life Sci 879(7-8):520-526

38. Shaner RL, Allegood JC, Park H, Wang E, Kelly S, Haynes CA, Sullards MC, Merrill AH Jr (2009) Quantitative analysis of sphingolipids for lipidomics using triple quadrupole and quadrupole linear ion trap mass spectrometers. J Lipid Res 50(8):1692-1707

39. Byrdwell WC, Perry RH (2006) Liquid chromatography with dual parallel mass spectrometry and (31)P nuclear magnetic resonance spectroscopy for analysis of sphingomyelin and 
dihydrosphingomyelin I. Bovine brain and chicken egg yolk. J Chromatogr A 1133(1-2):149-171

40. Geis-Asteggiante L, Lehotay SJ, Lightfield AR, Dutko T, Ng C, Bluhm L (2012) Ruggedness testing and validation of a practical analytical method for $>100$ veterinary drug residues in bovine muscle by ultrahigh performance liquid chromatography-tandem mass spectrometry. J Chromatogr A 1258:43-54

41. Evseenko D, Latour B, Richardson W, Corselli M, Sahaghian A, Cardinal S, Zhu Y, Chan R, Dunn B, Crooks GM (2013) Lysophosphatidic acid mediates myeloid differentiation within the human bone marrow microenvironment. PLoS One 8(5):e63718

42. Chen RF (1967) Removal of fatty acids from serum albumin by charcoal treatment. J Biol Chem 242(2):173-181
43. Jones BR, Schultz GA, Eckstein JA, Ackermann BL (2012) Surrogate matrix and surrogate analyte approaches for definitive quantitation of endogenous biomolecules. Bioanalysis 4(19):23432356

44. Chen S, Wu JT, Huang R (2012) Evaluation of surrogate matrices for standard curve preparation in tissue bioanalysis. Bioanalysis 4(21): 2579-2587

45. Bowen CL, Kehler J, Evans CA (2010) Development and validation of a sensitive and selective UHPLC-MS/MS method for simultaneous determination of both free and total eicosapentaeonic acid and docosahexenoic acid in human plasma. J Chromatogr B Anal Technol Biomed Life Sci 878(30):3125-3133 